Original article

\title{
Relating interferon regulatory factor 5 rs2004640 gene polymorphism to increased risk of systemic sclerosis in the patients: Russian Federation cohort
}

\author{
Mikhail Yu. Krylov, Lidia P. Ananieva, Irina A. Guseva \\ V.A. Nasonova Research Institute of Rheumatology, Moscow, Russia \\ Received 18 April 2019, Revised 28 January 2020, Accepted 22 April 2020
}

(C) 2019, Krylov M.Yu., Ananieva L.P., Guseva I.A.

(C) 2019, Russian Open Medical Journal

\begin{abstract}
Background - A number of studies confirmed a crucial role of type 1 interferon in pathophysiology of connective tissue diseases. Interferon regulatory factors (IRF) coordinate an expression of type 1 interferon, while interferon regulatory factor 5 (IRF5) gene was recently identified as causing predisposition to systemic lupus erythematosus and Sjögren syndrome.

The objective of our study was to identify possible association of IRF5 rs2004640 (G/T) single nucleotide polymorphism with systemic sclerosis (SSc).

Material and Methods-The study involved 236 individuals, including 105 patients with SSc diagnosis and 131 control individuals from Moscow region. The latter were healthy, unrelated to each other, their genders and ages were matched to those of SSc patients. Allelespecific real-time polymerase chain reaction (PCR) was used to study IFR5 rs2004640 polymorphism.

Results - We detected significantly higher percentage of IRF5 T-allele carriers in all patients (59.5\%), those with diffuse cutaneous SSc (67.3\%), patients with interstitial lung lesions (62.3\%), and those with positive titers of anti-topoisomerase I antibodies (66.3\%), compared with control group (46.2\%). The odds ratios (OR) were: $1.71(p=0.00), 2.40(p=0.0004), 1.93(p=0.002)$, and $2.30(p=0.0008)$, correspondingly.

Conclusion - The replacement of nucleotide G by T in the IRF5 rs2004640 gene polymorphism was associated with a predisposition to SSc. Our data implied an existence of a novel SSc pathogenetic pathway associated with important role of type 1 interferon in pathophysiology of connective tissue diseases.
\end{abstract}

Keywords: interferon, IRF5 gene polymorphism, systemic sclerosis, interstitial lung disease.

Cite as Krylov MYu, Ananieva LP, Guseva IA. Relating interferon regulatory factor 5 rs 2004640 gene polymorphism to increased risk of systemic sclerosis in the patients: Russian Federation cohort. Russian Open Medical Journal 2020; 9: e0410.

Correspondence to Mikhail Krylov. Email: mekry@yandex.ru.

\section{Introduction}

Systemic sclerosis (SSc) is a chronic multisystem connective tissue disorder characterized by immune system dysfunction associated with production of autoantibodies; early development of generalized vasculopathy; extensive deposition of collagen and other matrix proteins in connective tissues; and fibrosis of internal organs. SSC is linked to a higher mortality rate compared with other rheumatic diseases, such as rheumatoid arthritis (RA), systemic lupus erythematosus (SLE), or Sjögren syndrome (SS) [1]. The most common causes of mortality associated with SSc include interstitial lung disease (ILD) and pulmonary hypertension (PH). The exact etiology of SSc and accompanying ILD is currently unclear, but clinical and experimental data confirm multifactorial pathogenesis of this disease involving genetic and environmental factors [2]. Recent genetic studies confirmed that a number of candidate genes and their single nucleotide polymorphisms (SNPs) could be risk factors for SSc [3].

Type 1 interferon (IFN-1) is a central regulator of natural immunity, and its production is an ordinary physiological immune response to microbial infections. It stimulates various physiological processes: maturation of monocytes from dendritic cells, maturation of plasma cells, switching in immunoglobulin synthesis, activity of cytotoxic T cells and natural killer cells, and secretion of chemokines $[4,5]$. Studies involving microchips have shown an important role of IFN-1 in pathophysiology of SLE and SS [6, 7], as well as of other autoimmune disorders, such as dermatomyositis (DM), psoriasis (Ps), RA [8] and (as it was recently shown) SSc [9]. IRF5 is an important regulator of IFN-1 signal transmission. A largesample French case-control study established an association between IRF5 rs2004640 gene polymorphism and SSc, especially dcSSc (diffuse cutaneous systemic sclerosis) subtype [9]. In a Japanese study [10], the authors confirmed the association of IRF5 rs2004640 polymorphism with SSc, and also discovered additional associations of rs10954213 and rs2280714 polymorphisms with SSc, which were most pronounced in the patients with dcSSc phenotype and anti-topoisomerase I autoantibodies seropositivity (ATA+). In Dieude et al. study [11] of three SNPs in IRF5 rs3757385, rs2004640 and rs10954213 genes, the authors found two haplotypes designated as "R" (C-T-A) - a "risk haplotype" associated with dcSSc subtype of systemic sclerosis, and "P" (A-G- 
G) - a "protective haplotype". The haplotypes were associated with both cutaneous SSc subtypes and fibrosing alveolitis. Several other genome studies have also confirmed the association of IRF5 with SSc [12, 13]. Up to date, the role of IRF5 rs2004640 polymorphism in the Russian Federation population has not been studied in terms of its association with clinical and autoimmune phenotypes, as well as in terms of causing the patients' predisposition to SSc development.

An objective of our study was to investigate potential association of IRF5 rs2004640 (G/T) gene polymorphism with susceptibility of the Russian Federation population to SSC.

\section{Material and Methods}

\section{Study design}

Our case-control study involved 236 people; including 105 patients with SSc diagnosis ( 13 males, $12.4 \%$, and 92 females, $87.6 \%$ ) and a control group of 131 healthy, unrelated to each other individuals from Moscow region, their genders and ages matched to those of SSc patients. All SSc patients on the record at V.A. Nasonova Institute of Rheumatology clinic from September 2011 to January 2014 had an average age of $49.8 \pm 12.4 \mathrm{yr}$ and average disease duration of $11.2 \pm 9.3$ yr. All SSc patients meet 1980 American College of Rheumatology (ACR) criteria for the SSC classification [14]. Patients with typically manifest SSc represented the study group. All patients in the study group manifested at least three of five CREST syndromes (calcinosis, Raynaud's phenomenon, esophageal dysmotility, sclerodactyly, and telangiectasias). Based on medical documentation (diagnosis at discharge), all patients were stratified by their clinical phenotypes. None of the control group individuals had any autoimmune disorders.

\section{Computed tomography of the lungs}

Pulmonary fibrosis in the patients was established on the basis of characteristic pattern of the data obtained viaspiral computed tomography of the chest: presence of ILDwas interpreted as ILD(+).

\section{Immunological Assays}

Autoimmune phenotypes included patients with positive serum titers of antinuclear autoantibodies (ANA+), antitopoisomerase I antibodies (ATA+) or anti-centromere antibodies $(\mathrm{ACA}+)$. Indirect immunofluorescence on HEp-2 cells (II-HEp-2) with "IMMCO Diagnostics", USA, reagent kit was used to detect ANA and ACA. ATA was identified by enzyme-linked immunosorbent assay (ELISA) using "Orgentec" reagent kit (Germany). ANA and ATA titers $>1 / 160 \mathrm{U} / \mathrm{ml}$ and ATA $>25.0 \mathrm{U} / \mathrm{ml}$ were considered positive outcomes.

\section{Genotyping Assay}

Samples of venous blood were obtained from all participants. DNA was isolated from fresh or frozen blood samples using "GSgenetics" kit manufactured by "DNA Technology" (Moscow, Russia). We used allele-specific real-time PCR to study IFR5 rs2004640 polymorphism. Labeled primers and probes, amplification conditions were synthesized and developed by "SYNTOL" CJSC (Moscow, Russia). We have also used DT-96 amplifier by "DNA Technology" (Moscow, Russia). Ten percent of analyzed DNA samples from SSc patients were sequenced to confirm $100 \%$ match with genotyping outcomes.

V.A. Nasonova Institute of Rheumatology Ethics Committee approved the study, and written informed consent was obtained from all patients.

\section{Statistical analysis}

Differences in genotype frequency distribution between the patients and control individuals were assessed using $2 \times 2$ contingency tables and Fisher's exact test. The most common GG genotype was used as reference. Clinical phenotypes were presented as dichotomous variables. Our data on age and ailment duration were presented in the form: mean \pm standard deviation $(\mathrm{m} \pm \mathrm{SD})$, and percentages were analyzed. We used ANOVA post hoc test for analyzing the relationship among dichotomous variables and IRF5 gene polymorphism. The odds ratios (OR) and their $95 \%$ confidence intervals $(95 \% \mathrm{Cl})$ were calculated. Frequency distributions accordance Hardy-Weinberg law in the control group were tested using the chi-square $\left(\chi^{2}\right)$ test with one degree of freedom. A value of $p<0.05$ was considered statistically significant. Two-tailed p-values (Fisher's exact test) were computed for processing small-value variables. The analysis was performed using Statistica 6.1 software package (StatSoft Inc, Tulsa, USA).

\section{Results}

\section{Patient traits}

Demographics, along with clinical and immunological phenotypes of assessed SSc patients are presented in Table 1.

When using the chi-square $\left(\chi^{2}\right)$ test, distribution of genotype frequencies in the control group was consistent with HardyWeinberg law.

\section{IRF5G/T variants, and SSC clinical and serological phenotypes.}

We found significant differences in the distribution of IRF5 Tallele frequencies between SSc patients and control subjects: $\chi^{2}=8.1, p=0.017$ (Table 2). IRF5 T-allele frequency in SSc patients was $59.5 \%$ compared to $46.2 \%$ in control subjects [OR $1.71,95 \% \mathrm{Cl}$ (1.17-2.52), $p=0.004]$.

Table 1. Demographic and clinical phenotypes of systemic sclerosis patients

\begin{tabular}{lcc}
\hline Traits & SSc patients $n=105$ & Control $n=131$ \\
\hline Gender: Male /Female & $13 / 92$ & $17 / 114$ \\
Age, years, mean ( \pm SD) & $49.8 \pm 12.4$ & $51.3 \pm 13.6$ \\
Disease duration, years, mean ( \pm SD) & $11.2 \pm 9.3$ & - \\
$\leq 3$ years (M $\pm \delta)$ & $11(10.8)$ & - \\
$>3$ years (M $\pm \delta)$ & $91(89.2)$ & - \\
IcSSc $n(\%)$ & $53(51)$ & - \\
dcSSc $n(\%)$ & $52(49)$ & - \\
ILD $(+) n(\%)$ & $81(77)$ & - \\
ANA $(+) n(\%)$ & $88(84)$ & - \\
ATA $(+) n(\%)$ & $52(50)$ & - \\
ACA $(+) n(\%)$ & $17(15)$ & - \\
\hline
\end{tabular}

ICSSc, limited cutaneous SSc subtype; dcSSc, diffuse cutaneous SSc subtype; $\operatorname{ILD}(+)$, interstitial lung disease; $\mathrm{ANA}(+)$, antinuclear autoantibodies positivity; ATA(+), anti-topoisomerase I antibodies positivity; ACA(+), anticentromere antibodies positivity. 
Table 2. The distribution of genotypes and IRF5 allele frequencies in all SSc patients and in subgroups by clinical and serological phenotypes vs the control group.

\begin{tabular}{|c|c|c|c|c|c|c|c|}
\hline \multirow[t]{2}{*}{ IRF5 G/T gene } & \multicolumn{3}{|c|}{ Genotypes } & \multirow[t]{2}{*}{ Total } & \multirow[t]{2}{*}{ Frequency of T allele $n(\%)$} & \multirow[t]{2}{*}{ OR 95\%Cl } & \multirow[t]{2}{*}{$p$} \\
\hline & GG n (\%) & GT n (\%) & TT n (\%) & & & & \\
\hline Control & $39(29.8)$ & $63(48.1)$ & $29(22.1)$ & 131 & $121(46.2)$ & & \\
\hline IcSSc & $20(19.0)$ & $45(42.8)$ & $40(38.2)$ & 105 & 125 (59.5) & $1.71(1.17-2.52)$ & 0.004 \\
\hline dsSSc & $13(24.5)$ & $24(47.3)$ & $15(28.3)$ & 53 & 55 (51.9) & $1.26(0.78-2.03)$ & NS \\
\hline $\operatorname{ILD}(+)$ & $7(13.5)$ & $20(38.5)$ & $25(48.1)$ & 52 & $69(67.6)$ & $2.40(1.45-3.99)$ & $<0.001$ \\
\hline $\mathrm{ANA}(+)$ & $10(12.3)$ & $41(50.6)$ & $30(37.0)$ & 81 & $101(62.5)$ & $1.93(1.27-2.94)$ & 0.012 \\
\hline $\operatorname{ATA}(+)$ & $12(13.8)$ & $38(44.7)$ & $37(42.5)$ & 87 & $112(64.4)$ & $2.11(1.39-3.16)$ & $<0.001$ \\
\hline SSc & $4(7.8)$ & $26(51.0)$ & $21(41.1)$ & 51 & $68(66.7)$ & $2.30(1.40-3.81)$ & $<0.001$ \\
\hline
\end{tabular}

$\mathrm{p}$, patients vs control (for T-allele frequencies); statistically significant results are in bold; IcSSc, limited cutaneous SSc subtype; dcSSc, diffuse cutaneous SSc subtype; ILD(+), interstitial lung disease; ANA(+), antinuclear autoantibodies positivity; ATA(+), anti-topoisomerase I antibodies positivity; OR, odds ratio; $95 \% \mathrm{Cl}, 95 \%$ confidence interval; NS: not significant.

The IRF5 TT genotype was detected in $38.2 \%$ of SSc patients compared to $22.1 \%$ in the control group [OR $2.16,95 \% \mathrm{Cl}(1.18$ 3.99), $p=0.011]$. In the patients with SSc duration of over 3 years, the IRF5 T-allele and IRF5 TT genotype frequencies were higher compared to the control group $(63.1 \%$ and $46.2 \%, p=0.001 ; 41.3 \%$ and $20.1 \%, p=0.005$, respectively; these results are not presented in tables). There was no correlation of this polymorphism with gender and age.

Analysis of variance revealed statistically significant differences in the frequencies of the IRF5 $T$ allele between the groups of patients with dcSSc, ILD(+), ANA(+), ATA $(+)$ and the control group (Table 2). IRF5 T-allele carriers in the patient group were 2.4 times more susceptible to develop dcSSc, compared to carriers of the corresponding allele in the control group [OR $2.40,95 \% \mathrm{Cl}$ (1.453.99), $p=0.0004]$. In this SSc form, the frequency of the IRF5 TT genotype among patients was also increased, compared to control subjects [OR 3.26, 95\% Cl (1.55-6.80), $p=0.001$ ]. No reliable associations were found in the frequencies of the IRF5 $T$ allele between IcSSc (limited cutaneous systemic sclerosis) patients and control subjects.

Analysis of our data showed an association between IRF5 Tallele carriers and ILD(+). IRF5 T-allele carriers were 1.9 times more likely to develop SSc-related ILD (Table 2), compared with control. Homozygous mutant genotype frequency of the patients with this clinical phenotype was also significantly increased (Table 2). No significant differences were found in distribution of IRF5 T-allele frequencies in patient groups with ILD(+) vs. ILD.

IRF5 T-allele carrier frequency in the patients with ANA(+) phenotype was also significantly different from the corresponding frequency in the control group [OR 2.11, 95\% Cl (1.39-3.19), $\mathrm{p}=0.0001]$. Frequency of the IRF5 TT genotype among $\mathrm{ANA}(+)$ patients was also higher than in control (Table 2). However, we detected no differences when comparing IRF5 T-allele frequencies with homozygous genotype in ANA(-) group vs. control group. There were no significant differences in frequency distribution of IRF5 T allele vs. IRF5 TT genotype in the ANA(+) and ANA(-) patient groups.

IRF5 T-allele frequency was significantly higher in ATA(+) patients $(66.3 \%)$ vs. control $(p=0.0005)$. IRF5 T-allele carriers were 2.3 times more likely to form ATA(+) serological SSc phenotype in patients compared with control (Table 2). The frequencies of the IRF5 TT genotype also significantly differed between the patients and control subjects $(40.4 \%$ vs. $22.1 \%, p=0.012)$. We found no differences when comparing the frequencies of the IRF5 T allele between the ATA(-) group and control. There were no significant differences in frequency distributions of the $T$ allele and TT genotype in the patient groups with ATA $(+)$ and ATA(-).

Comparison of T-allele frequencies in $\mathrm{ACA}(+)$ subgroup vs. the controls was not feasible due to small $A C A(+)$ sample size (17 patients).

\section{Discussion}

Our results reliably confirmed the association between IRF5 rs2004640 polymorphism and SSc, therefore implying that we could consider it a SSc susceptibility gene. Our results were consistent with Dieude et al. [9] data arising from a large-sample study, in which IRF5 rs2004640 functional polymorphism was genotyped in 1641 subjects of French European Caucasian origin: an original set of 427 patients with SSc and 380 control subjects, and a replication set comprising of 454 patients with SSc and 380 control subjects. The authors showed that, in both samples, the IRF5 TT genotype was significantly more common in patients with SSc than in control subjects. For combined cohort, $\mathrm{OR}=1.58,95 \% \mathrm{Cl}=(1.18-2.11)$, $\mathrm{p}=0.002$. Analysis of SSc sample in France showed a significant association between homozygosity for the IRF5 $T$ allele and the presence of dcSSc, fibrosing alveolitis, ANA(+), and ATA positivity phenotype. The similarity in distribution of IRF5 rs2004640 alleles' and genotypes' polymorphism frequencies in the control groups of both studies (French and Russian) is worth mentioning. Current study demonstrated significant associations of IRF5 $T$ allele with various phenotypes: dcSSc subtype $(p=0.0004) ; \operatorname{ILD}(+), p=0.002$; $\operatorname{ANA}(+), p=0.0003$; and ATA(+), $p=0.0008$

An important role of three IRF5 SNP-haplotypes ( $r$ 10488631, rs2004640, and rs4728142) as susceptibility factors to SSc was reported in a multicenter study by Carmona et al. [15] with no specific associations with SSc phenotypes. Higher frequency of homozygous IRF5 TT genotype associated with IcSSc, pulmonary fibrosis, $\mathrm{ATA}(+)$ and $\mathrm{ACA}(+)$ was established for Chinese patients [16]. In the meta-analysis, $\mathrm{Xu}$ et al [17] showed the association of IRF5 rs2004640 polymorphism and rs7574865 polymorphism in signal transducer and activator of transcription (STAT4) genes with susceptibility to SSC, IcSSC and dcSSC variants. Another metaanalysis of eight SSc-associated SNP polymorphisms showed that solely IRF5 rs2004640, STAT4 rs7574865, IRAK1 rs1059702 and CTGF rs945 gene polymorphisms were associated with SSc and pulmonary fibrosis [18]. Since the first three polymorphisms were important regulatory control factors of innate immunity, and 
theCTGF gene, encoding the connective tissue growth factor, was involved in regulation of extracellular matrix synthesis, the results obtained by those authors confirmed the role of innate immunity and matrix components in pathogenesis of SSc-associated pulmonary fibrosis.

Lungs are among the targets of fibrosis process under SSc. We noticed a strong association between ILD(+) cases and IRF5 T allele, which was characteristic for $62.3 \%$ of the patients [OR $1.93, p=0.002$ ] and $37 \%$ of IRF5 $T$ T homozygous genotype carriers [OR 2.07, $p=0.028$ ]. In other words, it can be assumed that IRF5 rs2004640 gene polymorphism is a susceptibility factor for ILD development, although it is not a factor determining the severity of the ailment. A number of recent studies have shown that lung involvement in SSc is a multifactorial process, including sociodemographic and behavioral factors in addition to genetic causes [19].

The regulatory factor IRF5 coordinates the expression of IFN-1, which plays an important role in the pathophysiology of connective tissue diseases, such as SS, SLE and SSc [20]. Allele T of rs2004640 polymorphism creates a donor splicing site in one intron of the gene sequence transforming it in alternative $1 \mathrm{~B}$ exon.

Available literature presents evidence of occurrence of SSc-like phenotype and adverse events in the patients receiving interferon$\alpha$ or interferon- $\beta$ therapy in randomized and placebo-controlled trials [21]. It was noted that patients receiving active therapy in the form of subcutaneous injections of interferon- $\alpha$, had more severe lung function disorders and a tendency to skin progression, compared with the patients receiving placebo. Published data show the involvement of interferon- $\alpha$ and the IRF5 gene in pathophysiology of SSc and especially in ILD phenotype. Participation of the IFR5 gene as a genetic factor in predisposition to various autoimmune ailments seems to be important for understanding its role in interferon-dependent autoimmunity.

Previously, in a similar cohort of SSc patients, when studying the effect of rs7574865 (G/T) polymorphism of the STAT4 gene on the risk of developing SSc, we obtained similar results [22]. The STAT4 gene encodes the STAT-4 protein, which transmits signals from interleukins-12, 23, and interferon-1 into T-cells and monocytes. A significant association was shown for the rs7574865 T-allele polymorphism of the STAT4 gene with a predisposition to SSc and a number of its clinical variants: dsSSc, ILD(+), ATA (+). Thus, replacing G/T in rs2004640 and rs7574865 polymorphisms of the IRF5 and STAT4 genes lead to predisposition of mutant allele carriers of these genes to SSc. These data established that genetic polymorphisms can play a role in development of SSC and provide a new understanding of SSc pathogenesis.

\section{Conclusion}

Thus, the presented data exhibited reliable association between IRF5 rs2004640 gene polymorphism and SSc. We established that $\mathrm{T}$ allele was a risk factor causing the predisposition to dcSSc, ILD, and seropositivity to antitopoisomerase I antibodies. Our data implied a novel pathway for genetic factors participation in forming susceptibility to SSc and confirmed the IRF5 gene role in the genetic map of this disease.

\section{Limitations}

The data presented in a current study have a number of limitations related to the small sample size of the patients with
SSc, simultaneous case-control investigation, and require confirmation using large sample sizes of patients from different ethnic cohorts.

\section{Acknowledgments}

We thank Drs. O. Desinova, M. Starovopitova, and O. Koneva for patient recruitment, documentation, and collecting blood samples. Help of N. Konovalova and D. Varlamov, Russian Federal Research Institute for Agricultural Biotechnology, in designing real-time PCR is appreciated.

\section{Authors' contributions}

M.K. conducted genotyping assay; L.A. and I.G. recruited the patients and were involved in clinical studies; I.G. performed statistical analysis; M.K. and L.A. developed the study concept and design, coordinated the research and participated in draft manuscript preparation. All authors approved the final version of the manuscript.

\section{Funding}

This study received no external funding.

\section{Conflicts of Interest}

The authors declare no conflicts of interest.

\section{Ethical approva}

All procedures performed in our studies involving human participants were in accordance with the standards of the Ethics Committee at V.A. Nasonova Research Institute of Rheumatology (Moscow, Russia), as well as with 1964 Declaration of Helsinki and its later amendments, or comparable ethical standards.

\section{References}

1. Thomas E, Symmons DP, Brewster DH, Black RJ, Macfarlane GJ. National study of cause-specific mortality in rheumatoid arthritis, juvenile chronic arthritis, and other rheumatic conditions: a 20-year followup study. J Rheumatol 2003; 30(5): 958-965. https://pubmed.ncbi.nlm.nih.gov/12734889/.

2. Tan FK. Systemic sclerosis: the susceptible host (genetics and environment). Rheum Dis Clin North Am 2003; 29(2): 211-237. https://doi.org/10.1016/S0889-857X(03)00015-2.

3. Broen JC, Radstake TR, Rossato M. The role of genetics and epigenetics in the pathogenesis of systemic sclerosis. Nat Rev Rheumatol 2014; 10(11): 671-681. https://doi.org/10.1038/nrrheum.2014.128.

4. Lafyatis R, Marshak-Rothstein A. Toll-like receptors and innate immune responses in systemic lupus erythematosus. Arthritis Res Ther 2007; 9(6): 222. https://doi.org/10.1186/ar2321.

5. Honda K, Sakaguchi S, Nakajima C, Watanabe A, Yanai H, Matsumoto $\mathrm{M}$, et al. Selective contribution of IFN-alpha/beta signaling to the maturation of dendritic cells induced by double-stranded RNA or viral infection. Proc Natl Acad Sci U S A 2003; 100(19): 10872-10877. https://doi.org/10.1073/pnas.1934678100.

6. Baechler EC, Batliwalla FM, Karypis G, Gaffney PM, Ortmann, WA, Espe $\mathrm{KJ}$, et al. Interferon-inducible gene expression signature in periphera blood cells of patients with severe lupus. Proc Natl Acad Sci U S A 2003; 100(5): 2610-2615. https://doi.org/10.1073/pnas.0337679100.

7. Gottenberg JE, Cagnard N, Lucchesi C, Letourneur F, Mistou S, Lazure $\mathrm{T}$, et al. Activation of IFN pathways and plasmacytoid dendritic cell recruitment in target organs of primary Sjögren's syndrome. Proc Natl Acad Sci $U$ S A 2006; 103(8): 2770-2775. https://doi.org/10.1073/pnas.0510837103.

8. Baechler EC, Batliwalla FM, Reed AM, Peterson EJ, Gaffney PM, Moser $\mathrm{KL}$, et al. Gene expression profiling in human autoimmunity. Immunol 
$\operatorname{Rev} \quad 2006 ; \quad 210: \quad 120-137 . \quad$ https://doi.org/10.1111/j.01052896.2006.00367.x.

9. Dieudé P, Guedj M, Wipff J, Avouac J, Fajardy I, Diot E, et al. Association between the IRF5 rs2004640 functional polymorphism and systemic sclerosis: a new perspective for pulmonary fibrosis. Arthritis Rheum 2009; 60(1): 225-233. https://doi.org/10.1002/art.24183.

10. Ito I, Kawaguchi Y, Kawasaki A, Hasegawa M, Ohashi J, Hikami K, et al. Association of a functional polymorphism in the IRF5 region with systemic sclerosis in a Japanese population. Arthritis Rheum 2009; 60(6): 1845-1850. https://doi.org/10.1002/art.24600.

11. Dieude P, Dawidowicz K, Guedj M, Legrain $Y$, Wipff J, Hachulla E, et al. Phenotype-haplotype correlation of IRF5 in systemic sclerosis: role of 2 haplotypes in disease severity. J Rheumatol 2010; 37(5): 987-992. https://doi.org/10.3899/jrheum.091163.

12. Radstake TR, Gorlova O, Rueda B, Martin JE, Alizadeh BZ, PalominoMorales R, et.al. Genome-wide association study of systemic sclerosis identifies CD247 as a new susceptibility locus. Nat Genet 2010: 42(5): 426-429. https://doi.org/10.1038/ng.565.

13. Allanore $Y$, Saad $M$, Dieudé $P$, Avouac J, Distler JH, Amouyel $P$, et al. Genome-wide scan identifies TNIPI, PSORSICI, and RHOB as novel risk loci for systemic sclerosis. PLoS Genet 2011; 7(7): e1002091. https://doi.org/10.1371/journal.pgen.1002091.

14. Preliminary criteria for the classification of systemic sclerosis (scleroderma). Subcommittee for scleroderma criteria of the American Rheumatism Association Diagnostic and Therapeutic Criteria Committee.Arthritis Rheum 1980; 23(5): 581-590. https://doi.org/10.1002/art.1780230510.

15. Carmona FD, Martin JE, Beretta L, Simeón CP, Carreira PE, Callejas JL, et al. The systemic lupus erythematosus IRF5 risk haplotype is associated with systemic sclerosis. PLoS One 2013; 8(1): e54419. https://doi.org/10.1371/journal.pone.0054419.

16. Wang J, Yi L, Guo X, Liu M, Li H, Zou H, et al. Association of the IRF5 SNP rs2004640 with systemic sclerosis in Han Chinese. Int J Immunopathol Pharmacol 2014; 27(4): 635-638. https://doi.org/10.1177/039463201402700420.

17. Xu Y, Wang W, Tian Y, Liu J, Yang R. Polymorphisms in STAT4 and IRF5 increase the rask of systemic sclerosis: a meta-analysis. Int I Dermatol 2016; 55(4): 408-416. https://doi.org/10.1111/ijd.12839.

18. Zhao W, Yue X, Liu K, Zheng J, Huang R, Zou J, et al. The status of pulmonary fibrosis in systemic sclerosis is associated with IRF5, STAT4, IRAK1, and CTGF polymorphisms. Rheumatol Int 2017; 37(8): 13031310. https://doi.org/10.1007/s00296-017-3722-5.

19. McNearney TA, Reveille JD, Fischbach M, Friedman AW, Lisse JR, Goel $\mathrm{N}$, et al. Pulmonary involvement in systemic sclerosis: associations with genetic, serologic, sociodemographic, and behavioral factors. Arthritis Rheum 2007; 57(2): 318-326. https://doi.org/10.1002/art.22532.

20. Tan FK, Zhou X, Mayes MD, Gourh P, Guo X, Marcum C, et al. Signatures of differentially regulated interferon gene expression and vasculotrophism in the peripheral blood cells of systemic sclerosis patients. Rheumatology (Oxford) 2006; 45(6): 694-702. https://doi.org/10.1093/rheumatology/kei244.

21. Black CM, Silman AJ, Herrick Al, Denton CP, Wilson $\mathrm{H}$, Newman J, et al. Interferon-alpha does not improve outcome at one year in patients with diffuse cutaneous scleroderma: results of a randomized, doubleblind, placebo-controlled trial. Arthritis Rheum 1999; 42(2): 299-305. https://doi.org/10.1002/1529-0131(199902)42:2\%3C299::aidanr12\%3E3.0.co;2-r.

22. Krylov MYu, Ananyeva LP, Koneva OA, Starovoytova MN, Desinova OV, Ovsyannikova OB, et al. The influence of STAT4 rs7574865 (G/T) polymorphism on the risk of clinical and immunological phenotypes of systemic sclerosis in a Russian patient population: Results of a pilot study. Terapevticheskii Arkhiv 2017; 89(5): 20-25. Russian. https://doi.org/10.17116/terarkh201789520-25.

\section{Authors:}

Mikhail Yu. Krylov - MD, PhD, Senior Researcher, Laboratory of Immunology and Molecular Biology of Rheumatic Diseases, V.A. Nasonova Research Institute of Rheumatology, Moscow, Russia. https://orcid.org/0000-0002-9922-5124.

Lidia P. Ananieva - MD, DSc, Professor, Director of the Laboratory on Microcirculation and Inflammation, V.A. Nasonova Research Institute of Rheumatology, Moscow, Russia. https://orcid.org/0000-0002-3248-6426.

Irina A. Guseva- MD, PhD, Senior Researcher, Laboratory of Immunology and Molecular Biology of Rheumatic Diseases, V.A. Nasonova Research Institute of Rheumatology, Moscow, Russia. https://orcid.org/0000-00024906-7148. 